\title{
Bilateral patellar tendon rupture: a case report
}

\section{Henrique Costa Sousa*, Bárbara Choupina, Moisés Ventura, Ricardo Santos Pereira, André Costa, Paulo Jorge Carvalho}

\author{
Department of Orthopedics, Vila Nova de Gaia/Espinho Hospital Center, Vila Nova de Gaia, Portugal
}

Received: 28 December 2021

Accepted: 15 January 2022

\author{
*Correspondence: \\ Dr. Henrique Costa Sousa, \\ E-mail: henrique.acsousa@gmail.com
}

Copyright: (C) the author(s), publisher and licensee Medip Academy. This is an open-access article distributed under the terms of the Creative Commons Attribution Non-Commercial License, which permits unrestricted non-commercial use, distribution, and reproduction in any medium, provided the original work is properly cited.

\begin{abstract}
Bilateral patellar tendon rupture is a rare injury, usually associated with systemic disease or chronic medication side effect. Early diagnosis and prompt surgical repair are essential to achieve good functional outcomes. The authors report a case of a 44-year-old male with history of multiple urinary tract infection treated with fluoroquinolones who sustained spontaneous simultaneous bilateral patellar tendon rupture. Patient underwent bilateral transosseus tendon repair with autologous semitendinosus augmentation. Nine months after surgery, the patient was able to walk without pain and unassisted, had a normal range of motion in both knees and performed his daily activities without limitation. Surgical treatment of patellar tendon rupture is recommended to allow prevention of extensor mechanism disfunction sequelae. Transosseus tendon repair with autologous semitendinosus augment plasty is a valid surgical technique for treatment of this pathology with biomechanical and literature support.
\end{abstract}

Keywords: Patellar tendon rupture, Autologous semitendinous augmentation, Fluoroquinolones

\section{INTRODUCTION}

The patellar tendon is composed mainly of large collagen fibers ( $90 \%$ of type I collagen and $<10 \%$ of type III collagen); elastin, proteoglycans, and other non-collagen glycoproteins form the remainder of the ligament matrix. Vascular supply comes from the infrapatellar fat and the retinaculum; both ligament attachments are relatively avascular consisting in fibrocartilage. With a sagittal thickness ranging from 4 to $7 \mathrm{~mm}$, it's a strong structure which is not ruptured under normal physiological conditions, in fact a healthy patellar tendon rupture requires a force of 17.5 times the body weight. Patellar tendon rupture and extensor mechanism disruption generates major functional disability and gait disturbances. ${ }^{1,2}$

A bilateral patellar tendon rupture without significant trauma, even in the presence of predisposing factors is a rare injury. We describe a case of bilateral spontaneous simultaneous patellar tendon rupture.
The patient was informed that data concerning the case would be submitted for publication, and he consented.

\section{CASE REPORT}

A 44-year-old male patient with history of hypertension (treated with perindopril and bisoprolol), hypertriglyceridemia (treated with fenofibrate), overweight (Body mass index: $28.5 \mathrm{~kg} / \mathrm{m}^{2}$ ), obstructive apnea syndrome (under CPAP during night sleep), and past history of hypospadia with multiple urinary tract infections (UTI) treated with fluoroquinoles. There is no history of topic or systemic corticosteroids or steroid use. He presented to emergency department complaining of bilateral popping sensation followed by anterior knee pain and inability to walk that occurred while playing with his son in the garden. On physical examination bilateral joint effusion and infrapatellar gap were present, there was a complete loss of active knee extension on both sides. 
Plain radiographs showed bilateral superior patellar displacement. Ultrasound confirmed the diagnosis of complete proximal rupture of both patellar tendons, with a $2.5 \mathrm{~cm}$ inferior retraction. Magnetic resonance image of both knees confirmed important tendon degeneration and excluded associated knee injuries (Figures $1 \mathrm{~A}$ and B).
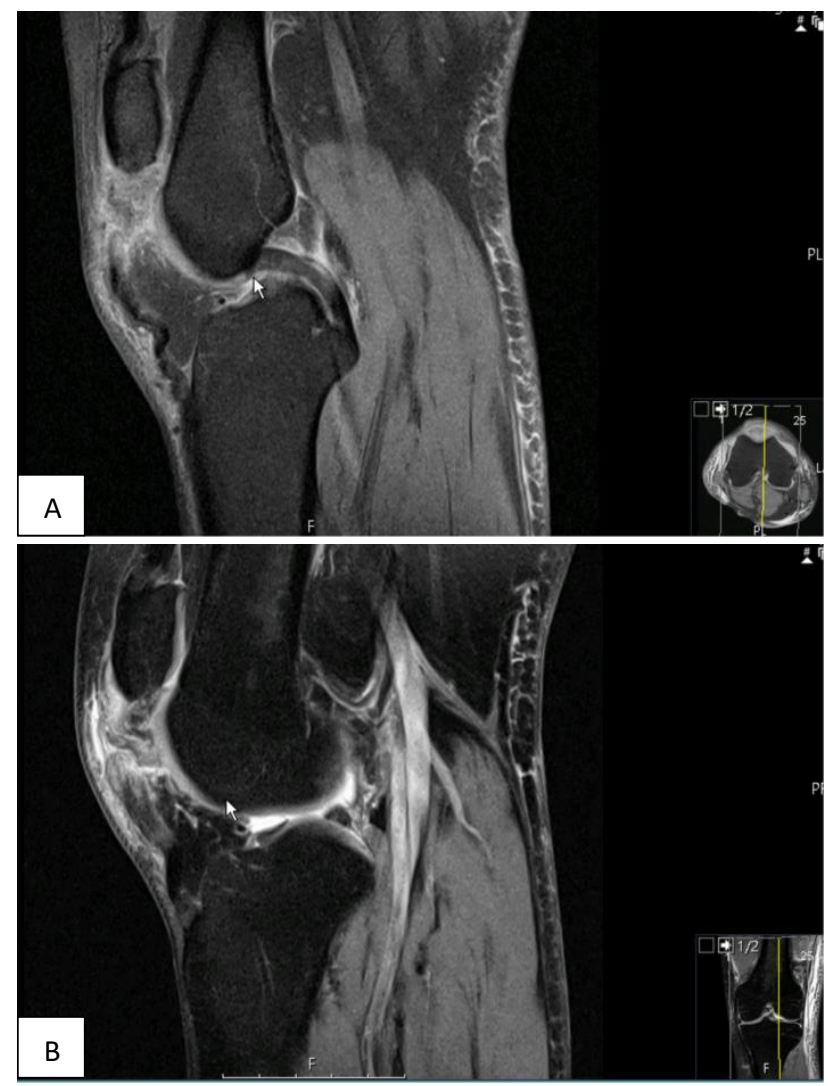

Figure 1 (A and B): T2 sagittal plan MRI of right and left knee with patellar tendon rupture.

Patient was admitted for surgical repair. Surgery underwent in supine position, tourniquet was applied in upper thigh, patellar tendon was approached via a midline incision. A proximal tendon injury was found in both knees that was associated with medial and lateral retinaculum rupture, marked tendon degeneration was evident (Figures 2 A-C). For transosseus repair tendon ends and patella inferior pole were debrided, the remaining distal tendon was whipstitched using two Krackow sutures with two Ethibond excel ${ }^{\circledR}$ (Johnson and Johnson) and channeled through three parallel drill holes $(2 \mathrm{~mm}$ diameter) made in the patella from distal to proximal to adapt the remaining tendon tissue (Figure 3B). Repair was reinforced with autologous semitendinosus augment plasty (Figure $3 \mathrm{C}$ ): semitendinosus tendon was harvested in standard fashion, then it was channeled through a transverse tibial tubercle drill hole and sutured to both the medial and lateral margins of the patellar tendon, and patella retinaculum. Medial and lateral retinaculum were repaired. The procedure was repeated in the other knee (Figure $3 \mathrm{~A}$ and $\mathrm{B}$ ). At the completion of the procedure, excellent stability of the patella, along with an adequate range of motion of both knee joints were achieved.
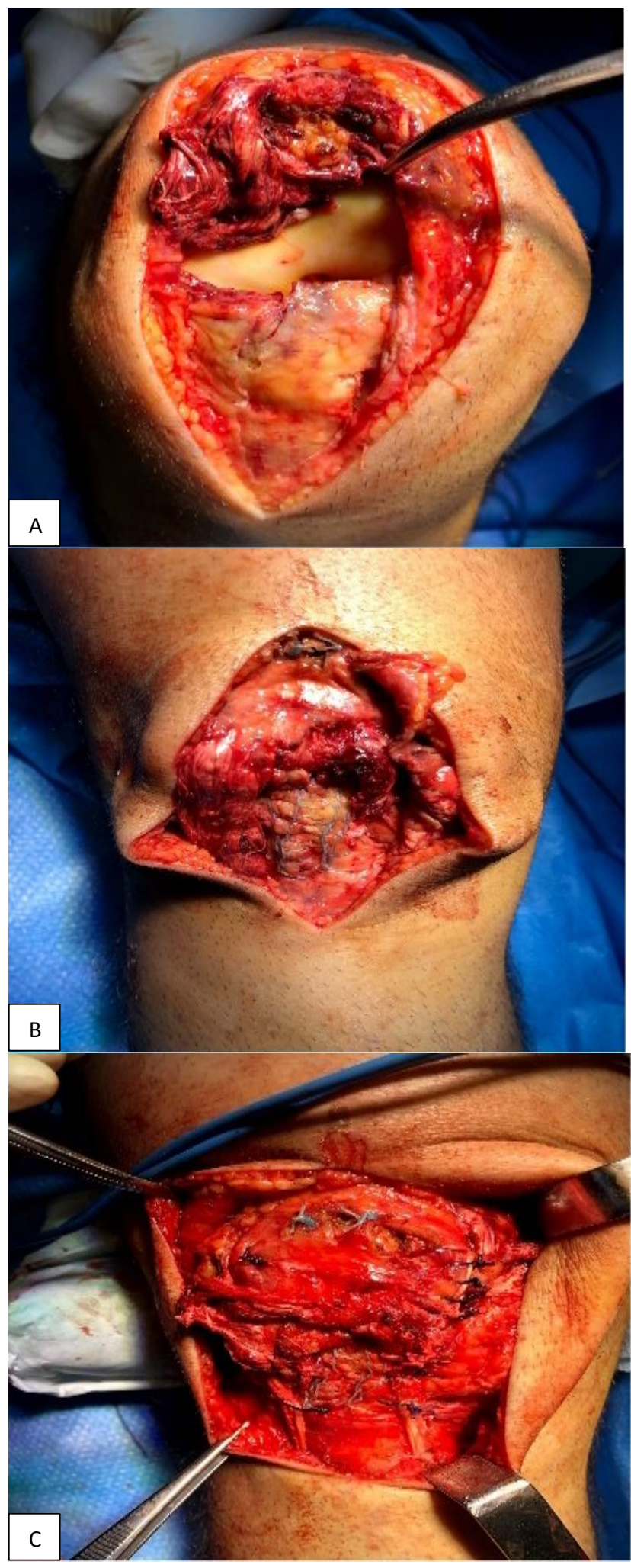

Figures 2 (A-C) Intra-operative right knee procedure of patellar injury, note tendon degeneration and fibroadipose tissue infiltration, re-insertion with transosseous sutures, and final result with semitendinous augmentation. 
At post-operative knee lateral view $\mathrm{X}$-ray with $30^{\circ}$ flexion the Insall-Salvatti ratio (ISR) of left knee was 1.41 and right knee 1.55 (Figure 4).

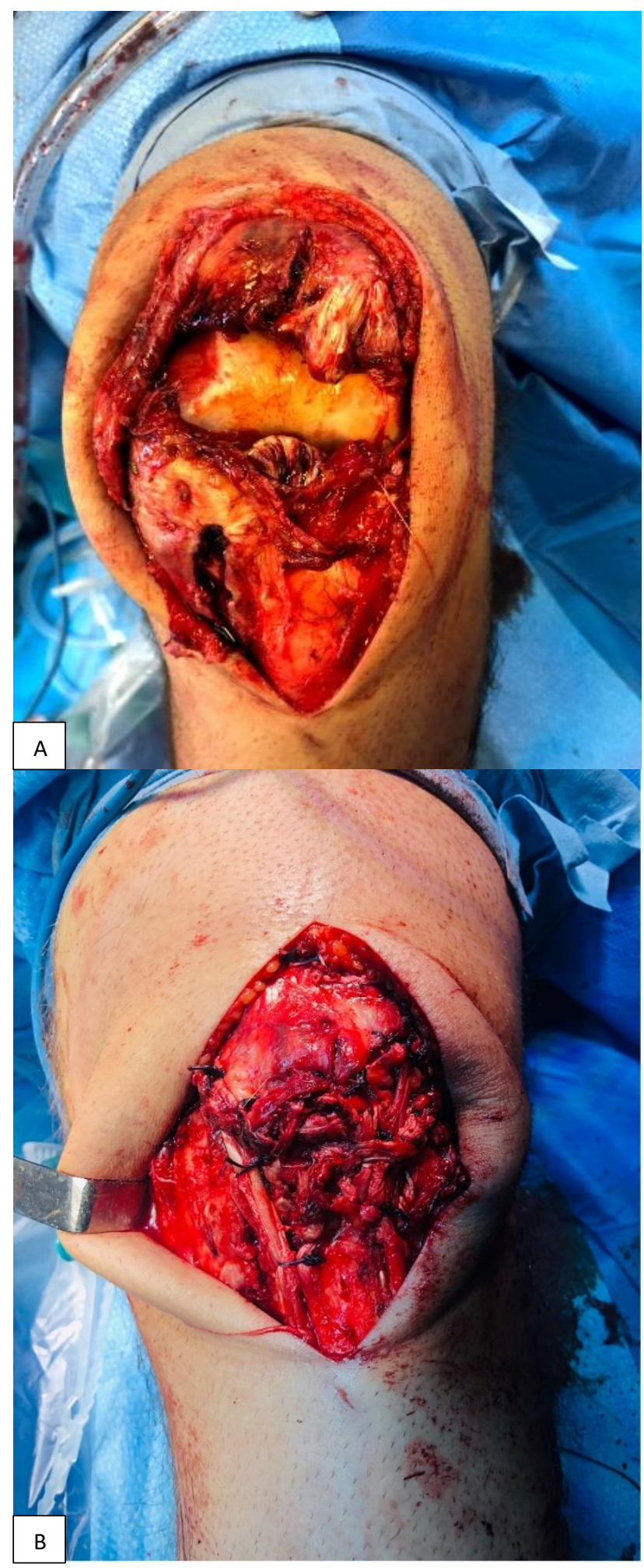

Figures 3 (A and B): Intra-operative left knee procedure showing patellar injury, note tendon degeneration and fibroadipose tissue infiltration, and final result with re-insertion with transosseous sutures and semitendinous augmentation.

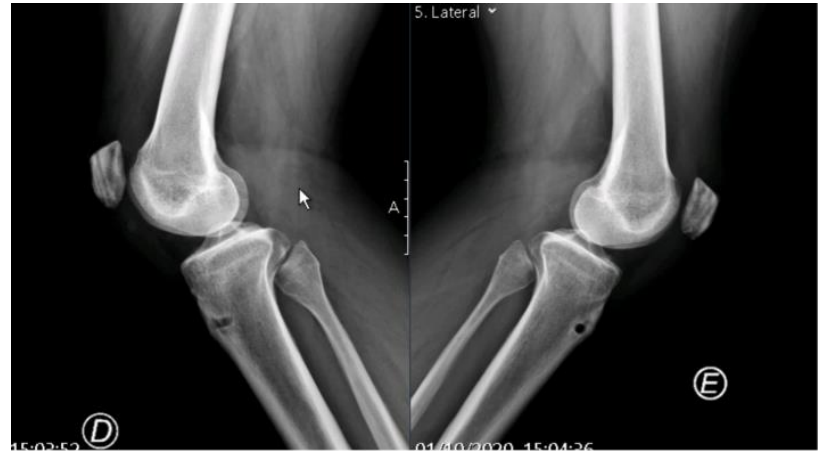

Figure 4: Lateral view right and left knee X-rays, showing patella alta.

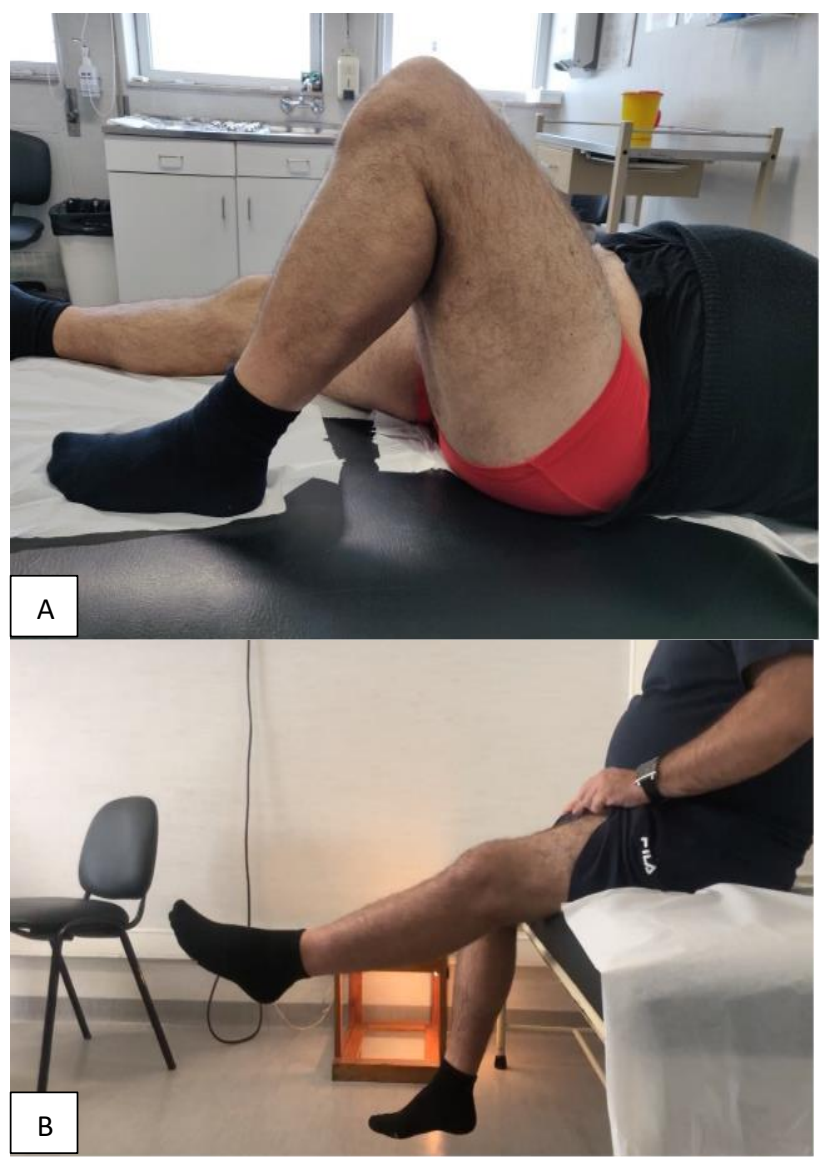

Figures 5 (A and B): Range of motion at 9-month follow-up.

Post-operatively patient was immobilized in inguinomalleolar brace locked in extension for 4 weeks, during this period he did at home-controlled range of motion exercises and weight bearing as tolerated with crutches was allowed, at this point the brace was discontinued. At 6 weeks progression to non-restricted weight bearing was started and underwent outpatient physical therapy for 4 months. At 3 months post-operatively he was walking unassisted, presenting a bilateral range of motion of $0^{\circ}$ to $120^{\circ}$ (Figures $5 \mathrm{~A}$ and $\mathrm{B}$ ) and was progressing in extension mechanism strength. He then progressively returned to baseline function and his activities of daily living. 
At one year follow-up functional outcome was evaluated: knee society score (KSS) final score 90-excellent; knee injury and osteoarthritis outcome score (KOOS) final score 81.5 (symptoms and stiffness $85.1 \%$; pain $77.78 \%$; daily living function $91.18 \%$; sports and recreational activities 65\%; quality of life 62.50\%); Tegner Lysholm knee scoring scale (TLS) final score 87-good; Kujala score (Anterior knee pain scale) final score $82 \%$.

Despite the radiographic finding of patella alta, the patient had no limitation of the extensor mechanism of either knee, neither complained of instability related symptoms.

\section{DISCUSSION}

Patellar tendon ruptures are typically unilateral and tend to occur in athletic adults under the age of $40 .{ }^{3}$ Patellar tendon rupture after minimal traumatic events or even in atraumatic conditions usually imply a weakened tendon with microstructural changes in the ligament substance. ${ }^{4}$ When bilateral, although it can be overuse tendinopathy, a spontaneous rupture of a degenerated tendon is usually the result of a systemic diseases or chronic medication side effect. Rheumatological diseases, diabetes, obesity, chronic renal failure, hyperparathyroidism, local and systemic corticosteroids, steroid use and antibiotics such as fluoroquinolones are all known risk factors for patellar tendon rupture. ${ }^{4,5}$ In this case the patient had history of hypospadia with multiple UTI treated with fluoroquinoles, which is increasingly being recognized as a cause of tendinitis and tendon rupture. ${ }^{6}$ Nevertheless, the estimated incidence of fluoroquinolones induced tendinopathy is only $0.14-0.4 \%$, being the most common site of involvement the achilles tendon; bilateral atraumatic patellar tendon rupture associated with fluoroquinolones use is exceedingly rare, with few cases described in the literature. ${ }^{4,8}$ The presumptive diagnosis of fluoroquinolone induced tendinopathy is bases on patient past medical history and the absence of any other obvious cause.

Diagnosis of patellar tendon rupture is usually based on clinical history and objective findings. A high energy trauma or risk factors for tendon rupture are usually present, typically patient complains of anterior knee pain and inability to walk that installed acutely after a popping sensation. On physical examination patients will have joint effusion, palpable infrapatellar soft-tissue defect, patella alta and complete or partial disruption of knee extensor mechanism, they will be unable to actively extend the knees or perform a straight leg raise. Bilateral rupture diagnosis can be more challenging as there is a healthy side lacking for comparison, late diagnosis results in treatment delay, which is associated with poorer outcomes specially if the gap between injury and surgery is $>2$ weeks. ${ }^{9}$ In addition to clinical findings diagnosis can be confirmed by complementary image: X-ray will show a patella alta, Ultrasound can confirm tendon disruption and MRI can provide further information about tendon quality, muscular atrophy or degeneration and associated injuries that can be identified or excluded before surgical intervention. ${ }^{10}$

Surgical treatment is mandatory to restore normal knee function. Early surgery is also associated with better outcomes, it can prevent decreased quadriceps strength and quadriceps contractures, which lead to tendon retraction and cause extensor dysfunction and diminished knee flexion, and also lessens the risk of late patellofemoral osteoarthritis., ${ }^{2,11}$ In these cases, chronic tendon degeneration implies poor tendon quality and healing capacity, therefore, contrary to pure traumatic injuries, primary repair, either by direct suture or transosseus reinsertion, will probably be insufficient and suture reinforcement must be considered. Furthermore, despite the good clinical outcome after acute repair, rerupture rates up to $50 \%$ have been described in monolateral injuries, therefore different augmentation strategies have been developed to decrease strain across the repair site. ${ }^{12,13}$ The semitendinosus reinforcement has been used by several authors with excellent functional outcomes, being it's ability in protection tendon repair supported by biomechanical studies. ${ }^{12,14}$ Others advantages of its use include their easy handling, strong nature, routine collection, minimal functional damage and no further surgery is required for implant removal. Alternatives to autograft include allograft, non-absorbable sutures augmentation, or internal splints like cerclage wire, the latter have the disadvantage of requiring a second surgery for hardware removal.

The ISR is the most widely used assessment of patellar height, it describes a constant relationship between the length of the patellar tendon and the patella. ${ }^{15}$ Patella alta, defined as a ISR $>1.2$, creates higher patellofemoral contact forces, which causes anterior knee pain, may be responsible for extensor lag and is a risk factor for patellar instability and patellofemoral osteoarthritis. ${ }^{15}$ In symptomatic cases the primary technique used to correct patella alta is a tibial tubercle osteotomy which distalizes the insertion of the tendon. In our case there is no preinjury X-ray to exclude constitutional patella alta. The patient has no limitation of the extensor mechanism of both knees nor does he complain of instability related symptoms or anterior knee pain. Therefore, it wasn't addressed.

\section{CONCLUSION}

Prompt diagnosis of patellar tendon rupture is mandatory to allow for early treatment and prevention of extensor mechanism disfunction sequelae. Transosseus tendon repair with autologous semitendinosus augment plasty is a commonly used surgical technique for treatment of this pathology with biomechanical and literature support that, in this case, achieved a satisfactory clinical outcome.

Funding: No funding sources

Conflict of interest: None declared

Ethical approval: Not required 


\section{REFERENCES}

1. Pengas IP, Assiotis A, Khan W. Adult native knee extensor mechanism ruptures. Injury. 2016;47:206570.

2. Savarese E, Bisicchia S, Amendola A. Bilateral spontaneous concurrent rupture of the patellar tendon in a healthy man: case report and review of the literature. Musculoskelet Surg. 2010;94(02):81-8.

3. Rose PS, Frassica FJ. Atraumatic bilateral patellar tendon rupture, A case report and review of the literature. J Bone Joint Surg Am. 2001;83$\mathrm{A}(09): 1382-6$.

4. Rosso F, Bonasia DE, Cottino U, Dettoni F, Bruzzone M, Rossi R. Patellar tendon: from tendinopathy to rupture. Asia-Pacific J Sports Med Arthrosc. Rehabil Technol. 2015;2(04):99-107.

5. Sibley T, Algren DA, Ellison S. Bilateral patellar tendon ruptures without predisposing systemic disease or steroid use: a case report and review of the literature. Am J Emerg Med. 2012;30:263-5.

6. Alves C, Mendes D, Marques FB. Fluoroquinolones and the risk of tendon injury: a systematic review and meta-analysis. Eur J Clin Pharmacol. 2019;75(10):1431-43.

7. Tsai WC, Yang YM. Fluoroquinolone associated tendinopathy. Chang Gung Med J. 2011;34:461-7.

8. Rosa B, Campos P, Barros A, Karmali S, Gonçalves R. Spontaneous bilateral patellar tendon rupture: case report and review of fluoroquinolone induced tendinopathy. Clin Case Rep. 2016;4(7):678-81.
9. Camarda L, D'arienzo A, Morello S. Bilateral ruptures of the extensor mechanism of the knee: a systematic review. J Orthop. 2017;14:445-53.

10. Von Glinski A, Yilmaz E, Rausch V, Königshausen M, Schildhauer TA, Seybold D et al. Semitendinosus autograft augmentation after bilateral patellar tendon re-rupture: a case report and technique note. Eur J Orthop Surg Traumatol. 2019;29(6):1347-53.

11. Juliato RH, Boschi LH, Juliato RF, Freitas AP, França AF, Bottura LB. Bilateral Atraumatic Patellar Ligament Rupture-Case Report. Rev Bras Ortop (Sao Paulo). 2019;54(2):223-7.

12. Maffulli N, Del Buono A, Loppini M, Denaro V. Ipsilateral hamstring tendon graft reconstruction for chronic patellar tendon ruptures: average 5.8-year follow-up. J Bone Joint Surg Am. 2013;95(17):e12316.

13. Gilmore JH, Clayton-Smith ZJ, Aguilar M. Reconstruction techniques and clinical results of patellar tendon ruptures: evidence today. Knee. 2015;22:148-55.

14. Mihalko WM, Vance M, Fineberg MJ. Patellar tendon repair with hamstring autograft: a cadaveric analysis. Clin Biomech (Bristol, Avon.) 2010;25(04):348-51.

15. Insall J, Salvati E. Patella position in the normal knee joint. Radiology. 1971;101:101-4.

Cite this article as: Sousa $\mathrm{HC}$, Choupina B, Ventura M, Pereira RS, Costa A, Carvalho PJ. Bilateral patellar tendon rupture: a case report. Int J Res Orthop 2022;8:251-5. 